\title{
Supplementary Materials for Dirichlet Process Mixture Models for Modeling and Generating Synthetic Versions of Nested Categorical Data
}

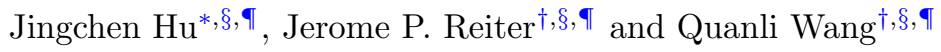

\section{Introduction}

Section 2 describes the full conditionals for the Gibbs sampler for both NDPMPM models. Section 3 presents a proof that the sampler for the NDPMPM with structural zeros gives draws from the posterior distribution of $\theta$ under the truncated model. Sections 4 to 7 present the results of the assessments of disclosure risks for the synthetic data illustrations in Section 4 of the main text. We describe the methodology for assessing risks in Section 4 and the computational methods in Section 5. We summarize the disclosure risk evaluations for the scenario without and with structural zeros in Section 6 and Section 7, respectively. Section 8 presents plots of point estimates versus the population values for both the synthetic and the original sample data, as described in Section 4 of the main text. Section 9 presents and compares results using an empirical prior and uniform prior distribution for the multinomial parameters in the no structural zeros simulation. Section 10 presents and compares results using $(F, S)=(50,50)$ and $(F, S)=(30,10)$ in the no structural zeros simulation.

\section{Full conditional distributions for MCMC samplers}

We present the full conditional distributions used in the Gibbs samplers for the versions of the NDPMPM with and without structural zeros. In both presentations, we assume common $\beta$ for all household-level clusters.

\subsection{NDPMPM without structural zeros}

\footnotetext{
*Department of Mathematics and Statistics, Vassar College, Box 27, Poughkeepsie, NY 12604 jihu@vassar.edu

${ }^{\dagger}$ Department of Statistical Science, Duke University, Durham, NC 27708-0251 jerry@stat.duke.edu

${ }^{\ddagger}$ Department of Statistical Science, Duke University, Durham, NC 27708-0251 quanli@stat.duke.edu

$\S$ National Science Foundation CNS-10-12141

INational Science Foundation SES-11-31897

" Arthur P. Sloan Foundation G-2015-2-166003
}

(C) 0000 International Society for Bayesian Analysis

DOI: 0000 
- Sample $G_{i} \in\{1, \ldots, F\}$ from a multinomial distribution with sample size one and probabilities

$$
\operatorname{Pr}\left(G_{i}=g \mid-\right)=\frac{\pi_{g}\left\{\prod_{k=p+1}^{q} \lambda_{g X_{i k}}^{(k)}\left(\prod_{j=1}^{n_{i}} \sum_{m=1}^{S} \omega_{g m} \prod_{k=1}^{p} \phi_{g m X_{i j k}}^{(k)}\right)\right\}}{\sum_{f=1}^{F} \pi_{f}\left\{\prod_{k=p+1}^{q} \lambda_{f X_{i k}}^{(k)}\left(\prod_{j=1}^{n_{i}} \sum_{m=1}^{S} \omega_{f m} \prod_{k=1}^{p} \phi_{f m X_{i j k}}^{(k)}\right)\right\}} .
$$

- Sample $M_{i j} \in\{1, \ldots, S\}$ given $G_{i}$ from a multinomial distribution with sample size one and probabilities

$$
\operatorname{Pr}\left(M_{i j}=m \mid-\right)=\frac{\omega_{G_{i} m} \prod_{k=1}^{p} \phi_{G_{i} m X_{i j k}}^{(k)}}{\sum_{s=1}^{S} \omega_{G_{i} s} \prod_{k=1}^{p} \phi_{G_{i} s X_{i j k}}^{(k)}} .
$$

- Set $u_{F}=1$. Sample $u_{g}$ from the Beta distribution for $g=1, \ldots, F-1$, where

$$
\begin{aligned}
\left(u_{g} \mid-\right) & \sim \operatorname{Beta}\left(1+\sum_{i=1}^{n} \mathbb{1}\left(G_{i}=g\right), \alpha+\sum_{f=g+1}^{F} \sum_{i=1}^{n} \mathbb{1}\left(G_{i}=f\right)\right) \\
\pi_{g} & =u_{g} \prod_{f<g}\left(1-u_{f}\right) .
\end{aligned}
$$

- Set $v_{g M}=1$. Sample $v_{g m}$ from the Beta distribution for $m=1, \ldots, S-1$, where

$$
\begin{aligned}
\left(v_{g m} \mid-\right) & \sim \operatorname{Beta}\left(1+\sum_{i=1}^{n} \mathbb{1}\left(M_{i j}=m, G_{i}=g\right), \beta+\sum_{s=m+1}^{S} \sum_{i=1}^{n} \mathbb{1}\left(M_{i j}=s, G_{i}=g\right)\right) \\
\omega_{g m} & =v_{g m} \prod_{s<m}\left(1-v_{g s}\right) .
\end{aligned}
$$

- Sample $\lambda_{g}^{(k)}$ from the Dirichlet distribution for $g=1, \ldots, F$, and $k=p+1, \ldots, q$, where

$$
\left(\lambda_{g}^{(k)} \mid-\right) \sim \operatorname{Dir}\left(a_{k 1}+\sum_{i \mid G_{i}=g}^{n} \mathbb{1}\left(X_{i k}=1\right), \ldots, a_{k d_{k}}+\sum_{i \mid G_{i}=g}^{n} \mathbb{1}\left(X_{i k}=d_{k}\right)\right) .
$$

- Sample $\phi_{g m}^{(k)}$ from the Dirichlet distribution for $g=1, \ldots, F, m=1, \ldots, S$ and $k=$ $1, \ldots, p$, where

$$
\left(\phi_{g m}^{(k)} \mid-\right) \sim \operatorname{Dir}\left(a_{k 1}+\sum_{\substack{G_{i}=g, i,\left.j\right|_{M}=m}}^{n, n_{i}} \mathbb{1}\left(X_{i j k}=1\right), \ldots, a_{k d_{k}}+\sum_{\substack{G_{i j}=m \\ i,\left.j\right|_{M_{i j}=m},}}^{n, n_{i}} \mathbb{1}\left(X_{i j k}=d_{k}\right)\right) .
$$

- Sample $\alpha$ from the Gamma distribution,

$$
(\alpha \mid-) \sim \operatorname{Gamma}\left(a_{\alpha}+F-1, b_{\alpha}-\sum_{g=1}^{F-1} \log \left(1-u_{g}\right)\right) .
$$


- Sample $\beta$ from the Gamma distribution,

$$
(\beta \mid-) \sim \operatorname{Gamma}\left(a_{\beta}+F *(S-1), b_{\beta}-\sum_{m=1}^{S-1} \sum_{g=1}^{F} \log \left(1-v_{g m}\right)\right) .
$$

\subsection{NDPMPM with structural zeros}

Let $\mathcal{X}^{0}$ include observations that are not admissible (they fail structural zero constraints). Let $\mathbf{G}^{0}$ and $\mathbf{M}^{0}$ be the latent class membership indicators for these records. Let the total number of households of size $h$ in $\mathcal{X}$ be written as $\left(n_{* h}+n_{0 h}\right)$, where $n_{0 h}$ is the number households of size $h$ generated in $\mathcal{X}^{0}$.

In each MCMC iteration, we have to sample $\left(n_{0 h},\left\{\left(\mathcal{X}_{i}^{0}, G_{i}^{0}, M_{i j}^{0}\right): i=1, \ldots, n_{0 h}, j=\right.\right.$ $1, \ldots, h\})$ for each $h \in \mathcal{H}$. We do so by means of a rejection sampler. To begin, we initialize $\mathcal{X}^{0}=\mathbf{G}^{0}=\mathbf{M}^{0}=\emptyset$ at each MCMC iteration. For each $h \in \mathcal{H}$, we repeat the following steps.

a. Set $t_{0}=0$. Set $t_{1}=0$.

b. Sample a value of $G_{i}$ from a multinomial distribution with sample size one and $\operatorname{Pr}\left(G_{i}=g \mid-\right) \propto \operatorname{Pr}\left(X_{i k}=h \mid G_{i}=g\right) \pi_{g}$, where $X_{i k}$ corresponds to the variable for household size.

c. For $j=1, \ldots, h$, sample a value of $M_{i j}$ from a multinomial distribution with sample size one and $\operatorname{Pr}\left(M_{i j}=m \mid-\right)=\omega_{G_{i m}}$.

d. Set $X_{i k}=h$. Sample remaining household level values and all individual level values using (1) and (2) from the main text. Let $\mathcal{X}_{i}^{0}$ be the simulated value.

e. If $\mathcal{X}_{i}^{0} \in \mathcal{S}_{h}$, let $t_{0}=t_{0}+1$ and $\mathcal{X}^{0}=\mathcal{X}^{0} \cup \mathcal{X}_{i}^{0}$. Similarly, let $\mathbf{G}^{0}=\mathbf{G}^{0} \cup G_{i}$ and $\mathbf{M}^{0}=\mathbf{M}^{0} \cup\left\{M_{i 1}, \ldots, M_{i h}\right\}$. Otherwise set $t_{1}=t_{1}+1$.

f. If $t_{1}<n_{* h}$, return to Step b. Otherwise set $n_{0 h}=t_{0}$.

- For observations in $\mathcal{X}^{*}$, sample $G_{i} \in\{1, \ldots, F\}$ from a multinomial distribution with sample size one and

$$
\operatorname{Pr}\left(G_{i}=g \mid-\right)=\frac{\pi_{g}\left\{\prod_{k=p+1}^{q} \lambda_{g X_{i k}}^{(k)}\left(\prod_{j=1}^{n_{i}} \sum_{m=1}^{S} \omega_{g m} \prod_{k=1}^{p} \phi_{g m X_{i j k}}^{(k)}\right)\right\}}{\sum_{f=1}^{F} \pi_{f}\left\{\prod_{k=p+1}^{q} \lambda_{f X_{i k}}^{(k)}\left(\prod_{j=1}^{n_{i}} \sum_{m=1}^{S} \omega_{f m} \prod_{k=1}^{p} \phi_{f m X_{i j k}}^{(k)}\right)\right\}} .
$$

- For observations in $\mathcal{X}^{*}$, sample $M_{i j} \in\{1, \ldots, S\}$ given $G_{i}$ from a multinomial distribution with sample size one and

$$
\operatorname{Pr}\left(M_{i j}=m \mid-\right)=\frac{\omega_{G_{i} m} \prod_{k=1}^{p} \phi_{G_{i} m X_{i j k}}^{(k)}}{\sum_{s=1}^{S} \omega_{G_{i} s} \prod_{k=1}^{p} \phi_{G_{i} s X_{i j k}}^{(k)}} .
$$


- Set $u_{F}=1$. Let $n_{0}=\sum_{h} n_{0 h}$. Sample $u_{g}$ from the Beta distribution for $g=1, \ldots, F-$ 1 , where

$$
\begin{aligned}
\left(u_{g} \mid-\right) & \sim \operatorname{Beta}\left(1+\sum_{i=1}^{n} \mathbb{1}\left(G_{i}=g\right), \alpha+\sum_{f=g+1}^{F} \sum_{i=1}^{n+n_{0}} \mathbb{1}\left(G_{i}=f\right)\right) \\
\pi_{g} & =u_{g} \prod_{f<g}\left(1-u_{f}\right) .
\end{aligned}
$$

- Set $v_{g M}=1$. Sample $v_{g m}$ from the Beta distribution for $m=1, \ldots, S-1$, where

$$
\begin{aligned}
\left(v_{g m} \mid-\right) & \sim \operatorname{Beta}\left(1+\sum_{i=1}^{n+n_{0}} \mathbb{1}\left(M_{i j}=m, G_{i}=g\right), \beta+\sum_{s=m+1}^{S} \sum_{i=1}^{n+n_{0}} \mathbb{1}\left(M_{i j}=s, G_{i}=g\right)\right) \\
\omega_{g m} & =v_{g m} \prod_{s<m}\left(1-v_{g s}\right) .
\end{aligned}
$$

- Sample $\lambda_{g}^{(k)}$ from the Dirichlet distribution for $g=1, \ldots, F$, and $k=p+1, \ldots, q$, where

$$
\left(\lambda_{g}^{(k)} \mid-\right) \sim \operatorname{Dir}\left(a_{k 1}+\sum_{i \mid G_{i}=g}^{n+n_{0}} \mathbb{1}\left(X_{i k}=1\right), \ldots, a_{k d_{k}}+\sum_{i \mid G_{i}=g}^{n+n_{0}} \mathbb{1}\left(X_{i k}=d_{k}\right)\right)
$$

- Sample $\phi_{g m}^{(k)}$ from the Dirichlet distribution for $g=1, \ldots, F, m=1, \ldots, S$ and $k=$ $1, \ldots, p$, where

$$
\left(\phi_{g m}^{(k)} \mid-\right) \sim \operatorname{Dir}\left(a_{k 1}+\sum_{\substack{G_{i}=g, i,\left.j\right|_{M_{i j}=m}}}^{n+n_{0}, n_{i}} \mathbb{1}\left(X_{i j k}=1\right), \ldots, a_{k d_{k}}+\sum_{\substack{G_{j}||_{M_{i j}=g,} \\ M_{i j}=m}}^{n+n_{0}, n_{i}} \mathbb{1}\left(X_{i j k}=d_{k}\right)\right)
$$

- Sample $\alpha$ from the Gamma distribution,

$$
(\alpha \mid-) \sim \operatorname{Gamma}\left(a_{\alpha}+F-1, b_{\alpha}-\sum_{g=1}^{F-1} \log \left(1-u_{g}\right)\right) .
$$

- Sample $\beta$ from the Gamma distribution,

$$
(\beta \mid-) \sim \operatorname{Gamma}\left(a_{\beta}+F *(S-1), b_{\beta}-\sum_{m=1}^{S-1} \sum_{g=1}^{F} \log \left(1-v_{g m}\right)\right) .
$$




\section{Proof that sampler converges to correct distribution in the truncated model}

In this section, we state and prove a result that ensures draws of $\theta$ from the sampler in Section 3 of the main text correspond to draws from the posterior distribution, $p\left(\theta \mid \mathcal{X}^{*}, T(\mathcal{S})\right)$. The proof follows the strategy in Manrique-Vallier and Reiter (2014). A key difference is that our MCMC algorithm proceeds separately for each $h$, generating households from the untruncated model until reaching $n_{* h}$ feasible households.

We begin by introducing notation for the augmented data, $\mathcal{X}$. Recall that $\mathcal{X}$ is a draw from a NDPMPM model without restrictions, i.e., all combinations of household and individual variables are allowed. We write $\mathcal{X}=\left(\mathcal{X}^{1}, \mathcal{X}^{0}\right)$, where $\mathcal{X}^{1}$ includes observations that are admissible (no structural zeros) and $\mathcal{X}^{0}$ includes observations that are not admissible (they fail structural zero constraints).

Each record in $\mathcal{X}$ is associated with a household-level and individual-level latent class assignment. Let $\mathbf{G}^{1}=\left(G_{1}^{1}, \ldots, G_{n}^{1}\right)$ and $\mathbf{M}^{1}=\left\{\left(M_{i 1}^{1}, \ldots, M_{i n_{i}}^{1}\right), i=1, \ldots, n\right\}$ include all the latent class assignments corresponding to households and individuals in $\mathcal{X}^{1}$. Let $\mathbf{G}^{0}=\left(G_{1}^{0}, \ldots, G_{n_{0}}^{0}\right)$ and $\mathbf{M}^{0}=\left\{\left(M_{i 1}^{0}, \ldots, M_{i n_{i}}^{0}\right), i=1, \ldots, n_{0}\right\}$ include all the latent class assignments corresponding to the $n_{0}$ cases in $\mathcal{X}^{0}$.

We seek to prove that one can obtain samples from $p\left(\theta \mid \mathcal{X}^{*}, T(\mathcal{S})\right)$ in the truncated NDPMPM from a sampler for $f\left(\theta, \mathbf{G}^{1}, \mathbf{G}^{0}, \mathbf{M}^{1}, \mathbf{M}^{0}, \mathcal{X}^{0},\left\{n_{0 h}: h \in \mathcal{H}\right\} \mid \mathcal{X}^{1}\right)$ under an untruncated NDPMPM model. Put formally, we want to prove the following theorem

Theorem 1: Let $\mathcal{X}^{*}$ comprise $n$ randomly sampled households from the truncated NDPMPM in (15) of the main text. Let $\mathcal{X}^{1}$ be generated from the NDPMPM without any concern over structural zeros, i.e., the model from Section 2 of the main text, so that no element of $\mathcal{X}^{1} \in \mathcal{S}$. Assume that $\mathcal{X}^{*}=\mathcal{X}^{1}$. Let the prior distribution on each $\left(n_{* h}+n_{0 h}\right)$ be $p\left(n_{* h}+n_{0 h}\right) \propto 1 /\left(n_{* h}+n_{0 h}\right)$. Then,

$$
\begin{gathered}
\int f\left(\theta, \mathbf{G}^{1}, \mathbf{G}^{0}, \mathbf{M}^{1}, \mathbf{M}^{0}, \mathcal{X}^{0},\left\{n_{0 h}\right\} \mid \mathcal{X}^{1}\right) \\
d \mathcal{X}^{0} d \mathbf{G}^{1} d \mathbf{G}^{0} d \mathbf{M}^{1} d \mathbf{M}^{0} d\left\{n_{0 h}\right\} \\
=p\left(\theta \mid \mathcal{X}^{*}, T(\mathcal{S})\right) .
\end{gathered}
$$

Here, we use integration signs rather than summation signs to simplify notation.

Before continuing with the proof, we note that the rejection sampling step in the algorithm for the truncated NDPMPM is equivalent to sampling each $n_{0 h}$ from negative binomial distributions. As evident in the proof, this distribution arises when one assumes a specific, improper prior distribution on $\left(n_{* h}+n_{0 h}\right)$ that is independent of $\theta$, namely $p\left(n_{* h}+n_{0 h}\right) \propto 1 /\left(n_{* h}+n_{0 h}\right)$ for each $h$. This improper prior distribution is used solely for computational convenience, as using other prior distributions would make the full conditional not negative binomial and hence complicate the sampling of $\mathcal{X}^{0}$. A similar strategy was used by Manrique-Vallier and Reiter (2014), who adapted the improper prior suggested by Meng and Zaslavsky (2002) and O'Malley and Zaslavsky (2008) for sampling from truncated distributions. 
Let $\mathbf{G}_{h}=\left\{G_{i}: n_{i}=h\right\}$ be the household level latent class assignments of size $h$ households and $\mathbf{M}_{h}=\left\{M_{i j}: n_{i}=h, j=1, \ldots, n_{i}\right\}$ be the individual level latent class assignments associated with members of size $h$ households. We split $\mathbf{G}_{h}$ into $\mathbf{G}_{h}^{1}$ and $\mathbf{G}_{h}^{0}$, representing the values for records in $\mathcal{X}^{1}$ and in $\mathcal{X}^{0}$ respectively. We similarly split $\mathbf{M}_{h}$ into $\mathbf{M}_{h}^{1}$ and $\mathbf{M}_{h}^{0}$. Let $\mathcal{X}_{h}^{1}=\left\{\mathcal{X}_{i}^{1}: n_{i}=h\right\}$, and let $\mathcal{X}_{h}^{0}=\left\{\mathcal{X}_{i}^{0}: n_{i}=h\right\}$. We emphasize that $\mathcal{X}^{1}$ is used in all iterations, whereas $\mathcal{X}^{0}$ is generated in each iteration of the MCMC sampler. Using this notation, we have

$$
\begin{aligned}
& \int f\left(\theta, \mathbf{G}^{1}, \mathbf{G}^{0}, \mathbf{M}^{1}, \mathbf{M}^{0}, \mathcal{X}^{0},\left\{n_{0 h}\right\} \mid \mathcal{X}^{1}\right) d \mathcal{X}^{0} d \mathbf{G}^{1} d \mathbf{G}^{0} d \mathbf{M}^{1} d \mathbf{M}^{0} d\left\{n_{0 h}\right\} \\
& \propto p(\theta) \prod_{h \in \mathcal{H}} \int f\left(\mathcal{X}_{h}^{1}, \mathbf{G}_{h}^{1}, \mathbf{M}_{h}^{1}, \mathcal{X}_{h}^{0}, \mathbf{G}_{h}^{0}, \mathbf{M}_{h}^{0}, n_{0 h} \mid \theta\right) \\
& d \mathbf{G}_{h}^{1} d \mathbf{M}_{h}^{1} d \mathcal{X}_{h}^{0} d \mathbf{G}_{h}^{0} d \mathbf{M}_{h}^{0} d n_{0 h} .
\end{aligned}
$$

Extending the generative model in Section 2, we view each $\mathcal{X}_{h}^{1}$ as a truncated sample from the households in $\mathcal{X}$ of size $h$. Let $\mathcal{A}_{h}^{1}$ and $\mathcal{A}_{h}^{0}$ be the set of row indexes of records in $\mathcal{X}_{h}^{1}$ and in $\mathcal{X}_{h}^{0}$, respectively. This implies for any given value of $\left(n_{* h}+n_{0 h}\right)$ that $f\left(\mathcal{X}_{h}^{1}, \mathbf{G}_{h}^{1}, \mathbf{M}_{h}^{1}, \mathcal{X}_{h}^{0}, \mathbf{G}_{h}^{0}, \mathbf{M}_{h}^{0} \mid \theta, n_{* h}+n_{0 h}\right)$

$$
\begin{aligned}
= & \left(\begin{array}{c}
n_{* h}+n_{0 h} \\
n_{0 h}
\end{array}\right) \prod_{i \in \mathcal{A}_{h}^{1}} \mathbb{1}\left\{\mathcal{X}_{i}^{1} \notin \mathcal{S}_{h}\right\} f\left(\mathcal{X}_{i}^{1} \mid G_{i}^{1}, \mathbf{M}_{i}^{1}, \theta\right) f\left(G_{i}^{1}, \mathbf{M}_{i}^{1} \mid \theta\right) \\
& \prod_{i \in \mathcal{A}_{h}^{0}} \mathbb{1}\left\{\mathcal{X}_{i}^{0} \in \mathcal{S}_{h}\right\} f\left(\mathcal{X}_{i}^{0} \mid G_{i}^{0}, \mathbf{M}_{i}^{0}, \theta\right) f\left(G_{i}^{0}, \mathbf{M}_{i}^{0} \mid \theta\right) .
\end{aligned}
$$

Substituting (3) in (2) and expanding the integrals, we have

$$
\begin{aligned}
& p(\theta) \prod_{h \in \mathcal{H}} \int f\left(\mathcal{X}_{h}^{1}, \mathbf{G}_{h}^{1}, \mathbf{M}_{h}^{1}, \mathcal{X}_{h}^{0}, \mathbf{G}_{h}^{0}, \mathbf{M}_{h}^{0}, n_{0 h} \mid \theta\right) d \mathbf{G}_{h}^{1} d \mathbf{M}_{h}^{1} d \mathcal{X}_{h}^{0} d \mathbf{G}_{h}^{0}, d \mathbf{M}_{h}^{0} d n_{0 h} \\
& \propto p(\theta) \prod_{h \in \mathcal{H}} \prod_{i \in \mathcal{A}_{h}^{1}} \int \mathbb{1}\left\{\mathcal{X}_{i}^{1} \notin \mathcal{S}_{h}\right\} f\left(\mathcal{X}_{i}^{1} \mid G_{i}^{1}, \mathbf{M}_{i}^{1}, \theta\right) f\left(G_{i}^{1} \mid \theta\right) \prod_{j=1}^{h} f\left(M_{i j}^{1} \mid \theta\right) d G_{i}^{1} d \mathbf{M}_{i}^{1} \\
& \prod_{h \in \mathcal{H}} \sum_{n_{0 h}=0}^{\infty} p\left(n_{* h}+n_{0 h}\right)\left(\begin{array}{c}
n_{* h}+n_{0 h} \\
n_{0 h}
\end{array}\right) \prod_{i \in \mathcal{A}_{h}^{0}} \int \mathbb{1}\left\{\mathcal{X}_{i}^{0} \in \mathcal{S}_{h}\right\} f\left(\mathcal{X}_{i}^{0} \mid G_{i}^{0}, \mathbf{M}_{i}^{0}, \theta\right) \\
& f\left(G_{i}^{0}, \mathbf{M}_{i}^{0} \mid \theta\right) d G_{i}^{0} d \mathbf{M}_{i}^{0} d \mathbf{X}_{\mathbf{i}}^{\mathbf{0}} \\
&=p(\theta) \prod_{h \in \mathcal{H}} \prod_{i \in \mathcal{A}_{h}^{1}} \int \mathbb{1}\left\{\mathcal{X}_{i}^{1} \notin \mathcal{S}_{h}\right\} f\left(\mathcal{X}_{i}^{1} \mid G_{i}^{1}, \mathbf{M}_{i}^{1}, \theta\right) f\left(G_{i}^{1} \mid \theta\right) \prod_{j=1}^{h} f\left(M_{i j}^{1} \mid \theta\right) d \mathbf{G}_{i}^{1} d \mathbf{M}_{i}^{1} \\
& \prod_{h \in \mathcal{H}} \sum_{n_{0 h}=0}^{\infty}\left(\begin{array}{c}
n_{* h}+n_{0 h}-1 \\
n_{0 h}
\end{array}\right)\left(\pi_{0 h}(\theta)\right)^{n_{0 h}}
\end{aligned}
$$




$$
\begin{aligned}
=p(\theta) & \prod_{h \in \mathcal{H}}\left(\prod_{i \in \mathcal{A}_{h}^{1}} \int \mathbb{1}\left\{\mathcal{X}_{i}^{1} \notin \mathcal{S}_{h}\right\} f\left(\mathcal{X}_{i}^{1} \mid G_{i}^{1}, \mathbf{M}_{i}^{1}, \theta\right) f\left(G_{i}^{1} \mid \theta\right) \prod_{j=1}^{h} f\left(M_{i j}^{1} \mid \theta\right) d \mathbf{G}_{i}^{1} d \mathbf{M}_{i}^{1}\right. \\
& \left.\left(1-\pi_{0 h}(\theta)\right)^{-n_{* h}}\right)
\end{aligned}
$$

From (15) in the main text, this expression is equivalent to $p(\theta) \prod_{i=1}^{n} p\left(\mathcal{X}_{i}^{*} \mid \theta\right)$ when $\mathcal{X}^{*}=\mathcal{X}^{1}$, as desired.

Thus, we can obtain samples from the posterior distribution $p\left(\theta \mid \mathcal{X}^{*}, T(\mathcal{S})\right)$ in the truncated NDPMPM model from the sampler for $f\left(\theta, \mathbf{G}^{*}, \mathbf{G}^{0}, \mathbf{M}^{*}, \mathbf{M}^{0}, \mathcal{X}^{0},\left\{n_{0 h}\right\} \mid \mathcal{X}^{*}\right)$ under the unrestricted NDPMPM model.

\section{Disclosure risk measures}

When synthesizing entire household compositions (but keeping household size distributions fixed), it is nonsensical for intruders to match the proposed synthetic datasets to external files, since there is no unique mapping of the rows (individuals) in the synthetic datasets $\mathbf{Z}$ to the rows in the original data $\mathbf{D}$, nor unique mapping of the households in $\mathbf{Z}$ to the households in $\mathbf{D}$ (except for household sizes with $n_{i}=1$ ). We therefore consider questions of the form: can intruders accurately infer from $Z$ that some individual or entire household with a particular set of data values is in the confidential data? When the combination of values is unique in the population (or possibly just the sample), this question essentially asks if intruders can determine whether or not a specific individual or household is in $D$ (Hu et al., 2014).

To describe the disclosure risk evaluations, we follow the presentation of $\mathrm{Hu}$ et al. (2014). We consider two possible attacks on Z Z, namely (i) the intruder seeks to learn whether or not someone with a particular combination of the $p$ individual-level variables and the $q$ household-level variables is in $\mathbf{D}$, and (ii) an intruder seeks to learn whether or not an entire household with a particular combination of household-level and individuallevel characteristics is in $\mathbf{D}$. For the first scenario, we assume that the intruder knows the values in $\mathbf{D}$ for all individuals but the target individual, say individual $i j$. We use $\mathbf{D}_{-i j}$ to denote the data known to the intruder. For the second scenario, we assume that the intruder knows the values in $D$ for all households but the target house, say household $i$. We use $\mathbf{D}_{-i}$ to denote the data known to the intruder. In many cases, assuming the intruder knows $\mathbf{D}_{-i j}$ or $\mathbf{D}_{-i}$ is conservative; for example, in random samples from large populations intruders are unlikely to know $N-1$ individuals or $n-1$ households selected in the sample. We adopt this strong assumption largely to facilitate computation. Risks deemed acceptable under this assumption should be acceptable for weaker intruder knowledge. We note that assuming the intruder knows all records but one is related to, but quite distinct from, the assumptions used in differential privacy (Dwork, 2006).

Let $T_{i j}$ or $T_{i}$ be the random variable corresponding to the intruder's guess about the true values of the target. Let $t$ generically represent a possible guess at the target, where for simplicity of notation we use a common notation for individual and household 
targets. Let $\mathcal{I}$ represent any information known by the intruder about the process of generating $Z$, for example meta-data indicating the values of $F, S$ and $\left(a_{\alpha}, b_{\alpha}, a_{\beta}, b_{\beta}\right)$ for the NDPMPM synthesizer.

For the first type of attack, we assume the intruder seeks the posterior probability,

$$
\begin{aligned}
\rho_{i j}^{t}=p\left(T_{i j}=t \mid \mathbf{Z}, \mathbf{D}_{-i j}, \mathcal{I}\right) & =\frac{p\left(\mathbf{Z} \mid T_{i j}=t, \mathbf{D}_{-i j}, \mathcal{I}\right) p\left(T_{i j}=t \mid \mathbf{D}_{-i j}, \mathcal{I}\right)}{\sum_{t \in \mathcal{U}} p\left(\mathbf{Z} \mid T_{i j}=t, \mathbf{D}_{-i j}, \mathcal{I}\right) p\left(T_{i j}=t \mid \mathbf{D}_{-i j}, \mathcal{I}\right)}(4) \\
& \propto p\left(\mathbf{Z} \mid T_{i j}=t, \mathbf{D}_{-i j}, \mathcal{I}\right) p\left(T_{i j}=t \mid \mathbf{D}_{-i j}, \mathcal{I}\right),
\end{aligned}
$$

where $\mathcal{U}$ represents the universe of all feasible values of $t$. Here, $p\left(\mathbf{Z} \mid T_{i j}=t, \mathbf{D}_{-i j}, \mathcal{I}\right)$ is the likelihood of generating the particular set of synthetic data given that $t$ is in the confidential data and whatever else is known by the intruder. The $p\left(T_{i j}=t \mid \mathbf{D}_{-i j}, \mathcal{I}\right)$ can be considered the intruder's prior distribution on $T_{i j}$ based on $\left(\mathbf{D}_{-i j}, \mathcal{I}\right)$.

As described in $\mathrm{Hu}$ et al. (2014), intruders can use $p\left(T_{i j}=t \mid \mathbf{Z}, \mathbf{D}_{-i j}, \mathcal{I}\right)$ to take guesses at the true value $t_{i j}$. For example, the intruder can find the $t$ that offers the largest probability, and use that as a guess of $t_{i j}$. Similarly, agencies can use $p\left(T_{i j}=t \mid\right.$ $\left.\mathbf{Z}, \mathbf{D}_{-i j}, \mathcal{I}\right)$ in disclosure risk evaluations. For example, for each $t_{i j} \in \mathbf{D}$, they can rank each $t$ by its associated value of $p\left(T_{i j}=t \mid \mathbf{Z}, \mathbf{D}_{-i j}, \mathcal{I}\right)$, and evaluate the rank at the truth, $t=t_{i j}$. When the rank of $t_{i j}$ is high (close to 1 , which we define to be the rank associated with the highest probability), the agency may deem that record to be at risk under the strong intruder knowledge scenario. When the rank of $t_{i j}$ is low (far from 1), the agency may deem the risks for that record to be acceptable.

When $\mathcal{U}$ is very large, computing the normalizing constant in (4) is impractical. To facilitate computation, we follow $\mathrm{Hu}$ et al. (2014) and consider as feasible candidates only those $t$ that differ from $t_{i j}$ in one variable, along with $t_{i j}$ itself; we call this space $\mathcal{R}_{i j}$. Restricting to $\mathcal{R}_{i j}$ can be conceived as mimicking a knowledgeable intruder who searches in spaces near $t_{i j}$. As discussed by $\mathrm{Hu}$ et al. (2014), restricting support to $\mathcal{R}_{i j}$ results in a conservative ranking of the $t \in \mathcal{R}_{i j}$, in that ranks determined to be acceptably low when using $\mathcal{R}_{i j}$ also are acceptably low when using $\mathcal{U}$.

For $T_{i}$, we use a similar approach to risk assessment. We compute

$$
\rho_{i}^{t}=p\left(T_{i}=t \mid \mathbf{Z}, \mathbf{D}_{-i}, \mathcal{I}\right) \propto p\left(\mathbf{Z} \mid T_{i}=t, \mathbf{D}_{-i}, \mathcal{I}\right) p\left(T_{i}=t \mid \mathbf{D}_{-i}, \mathcal{I}\right)
$$

We consider only $t$ that differ from $t_{i}$ in either (i) one household-level variable for the entire household or (ii) one individual-level variable for one household member, along with $t_{i}$ itself; we call this space $\mathcal{R}_{i}$.

\section{Computational methods for risk assessment with the NDPMPM model}

We describe the computational methods for computing (6) in detail. Methods for computing (5) are similar. 
For any proposed $t$, let $\mathbf{D}_{i}^{t}=\left(T_{i}=t, \mathbf{D}_{-i}\right)$ be the plausible confidential dataset when $T_{i}=t$. Because each $\mathbf{Z}^{(l)}$ is generated independently, we have

$$
P\left(\mathbf{Z} \mid \mathbf{D}_{i}^{t}, \mathcal{I}\right)=\prod_{l=1}^{L} P\left(\mathbf{Z}^{(l)} \mid \mathbf{D}_{i}^{t}, \mathcal{I}\right)
$$

Hence, we need to compute each $P\left(\mathbf{Z}^{(l)} \mid \mathbf{D}_{i}^{t}, \mathcal{I}\right)$.

Let $\Theta=\{\pi, \omega, \lambda, \phi\}$ denote parameters from a NDPMPM model. We can write $P\left(\mathbf{Z}^{(l)} \mid \mathbf{D}_{i}^{t}, \mathcal{I}\right)$ as

$$
P\left(\mathbf{Z}^{(l)} \mid \mathbf{D}_{i}^{t}, \mathcal{I}\right)=\int p\left(\mathbf{Z}^{(l)} \mid \mathbf{D}_{i}^{t}, \mathcal{I}, \Theta\right) p\left(\Theta \mid \mathbf{D}_{i}^{t}, \mathcal{I}\right) d \Theta .
$$

To compute (8), we could sample many values of $\Theta$ that could have generated $\mathbf{Z}^{(l)}$; that is, we could sample $\Theta^{(r)}$ for $r=1, \ldots, R$. For each $\Theta^{(r)}$, we compute the probability of generating the released $\mathbf{Z}^{(l)}$. We then average these probabilities over the $R$ draws of $\Theta$.

Conceptually, to draw $\Theta$ replicates, we could re-estimate the NDPMPM model for each $\mathbf{D}_{i}^{t}$. This quickly becomes computationally prohibitive. Instead, we suggest using the sampled values of $\Theta$ from $p(\Theta \mid \mathbf{D})$ as proposals for an importance sampling algorithm. To set notation, suppose we seek to estimate the expectation of some function $g(\Theta)$, where $\Theta$ has density $f(\Theta)$. Further suppose that we have available a sample $\left(\Theta^{(1)}, \ldots, \Theta^{(R)}\right)$ from a convenient distribution $f^{*}(\Theta)$ that slightly differs from $f(\Theta)$. We can estimate $E_{f}(g(\Theta))$ using

$$
E_{f}(g(\Theta)) \approx \sum_{r=1}^{R} g\left(\Theta^{(r)}\right) \frac{f\left(\Theta^{(r)}\right) / f^{*}\left(\Theta^{(r)}\right)}{\sum_{r=1}^{R} f\left(\Theta^{(r)}\right) / f^{*}\left(\Theta^{(r)}\right)} .
$$

Let $t_{i}^{*(l)}$ be the $i$ th household's values of all variables, including household-level and individual-level variables, in synthetic dataset $\mathbf{Z}^{(l)}$, where $i=1, \ldots, n$ and $l=1, \ldots, L$. For each $\mathbf{Z}^{(l)}$ and any proposed $t$, we define the $g(\Theta)$ in (9) to equal $c P\left(\mathbf{Z}^{(l)} \mid \mathbf{D}_{i}^{t}, \mathcal{I}\right)$. We approximate the expectation of each $g(\Theta)$ with respect to $f(\Theta)=f\left(\Theta \mid \mathbf{D}_{i}^{t}, \mathcal{I}\right)$. In doing so, for any sampled $\Theta^{(r)}$ we use

$g\left(\Theta^{(r)}\right)=P\left(\mathbf{Z}^{(l)} \mid \mathbf{D}_{i}^{t}, \mathcal{I}, \Theta^{(r)}\right)=\prod_{i=1}^{n}\left(\sum_{g=1}^{F} \pi_{g}^{(r)}\left\{\prod_{k=p+1}^{p+q} \lambda_{g t_{i k}^{*(l)}}^{(k)(r)}\left(\prod_{j=1}^{n_{i}} \sum_{m=1}^{S} \omega_{g m}^{(r)} \prod_{k=1}^{p} \phi_{g m t_{i j k}^{*(l)}}^{(k)(r)}\right)\right\}\right)$.

We set $f^{*}(\Theta)=f(\Theta \mid \mathbf{D}, \mathcal{I})$, so that we can use $R$ draws of $\Theta$ from its posterior distribution based on $\mathbf{D}$. Let these $R$ draws be $\left(\Theta^{(1)}, \ldots, \Theta^{(R)}\right)$. We note that one could use any $\mathbf{D}_{i}^{t}$ to obtain the $R$ draws, so that intruders can use similar importance sampling computations. As evident in (1), (2), (3) and (4) in the main text, the only differences in the kernels of $f(\Theta)$ and $f^{*}(\Theta)$ include (i) the components of the likelihood associated with record $i$ and (ii) the normalizing constant for each density. Let 
$\mathbf{t}=\left\{\left(c_{p+1}, \ldots, c_{p+q}\right),\left(c_{j 1}, \ldots, c_{j p}\right), j=1, \ldots, n_{i}\right\}$, where each $c_{k} \in\left(1, \ldots, d_{k}\right)$, be a guess at $T_{i}$, for household-level and individual-level variables respectively. After computing the normalized ratio in (9) and canceling common terms from the numerator and denominator, we are left with $P\left(\mathbf{Z}^{(l)} \mid \mathbf{D}_{i}^{t}, \mathcal{I}\right)=\sum_{r=1}^{R} p_{r} q_{r}$ where

$$
\begin{aligned}
& p_{r}=\prod_{i=1}^{n}\left(\sum_{g=1}^{F} \pi_{g}^{(r)}\left\{\prod_{k=p+1}^{p+q} \lambda_{g t_{i k}^{*(l)}}^{(k)(r)}\left(\prod_{j=1}^{n_{i}} \sum_{m=1}^{S} \omega_{g m}^{(r)} \prod_{k=1}^{p} \phi_{g m t_{i j k}^{*(l)}}^{(k)(r)}\right)\right\}\right) \\
& q_{r}=\frac{\frac{\sum_{g=1}^{F} \pi_{g}^{(r)}\left\{\prod_{k=p+1}^{p+q} \lambda_{g c}^{(k)(r)}\left(\prod_{j=1}^{n_{i}} \sum_{m=1}^{S} \omega_{g m}^{(r)} \prod_{k=1}^{p} \phi_{g m e}^{(k)(r)}\right)\right\}}{\sum_{g=1}^{F} \pi_{g}^{(r)}\left\{\prod_{k=p+1}^{p+q} \lambda_{g t_{i k}(r)}^{(k)(r)}\left(\prod_{j=1}^{n_{i}} \sum_{m=1}^{S} \omega_{g m}^{(h)} \prod_{k=1}^{p} \phi_{g m t_{i j k}}^{(k)(r)}\right)\right\}}}{\sum_{u=1}^{R}\left(\frac{\sum_{g=1}^{F} \pi_{g}^{(u)}\left\{\prod_{k=p+1}^{p+q} \lambda_{g g_{k}}^{(k)(u)}\left(\prod_{j=1}^{n_{i}} \sum_{m=1}^{S} \omega_{g m}^{(u)} \prod_{k=1}^{p} \phi_{g m e}^{(k)(u)}\right)\right\}}{\sum_{g=1}^{F} \pi_{g}^{(u)}\left\{\prod_{k=p+1}^{p+q} \lambda_{g t_{i k}}^{(k)(u)}\left(\prod_{j=1}^{n_{i}} \sum_{m=1}^{S} \omega_{g m}^{(u)} \prod_{k=1}^{p} \phi_{g m t_{i j k}}^{(k)(u)}\right)\right\}}\right)} .
\end{aligned}
$$

We repeat this computation for each $\mathbf{Z}^{(l)}$, plugging the $L$ results into (7).

Finally, to approximate $\rho_{i}^{t}$, we compute (7) for each $t \in \mathcal{R}_{i}$, multiplying each resulting value by its associated $P\left(T_{i}=t \mid \mathbf{D}_{-i}, \mathcal{I}\right)$. In what follows, we presume an intruder with a uniform prior distribution over the support $t \in \mathcal{R}_{i}$. In this case, the prior probabilities cancel from the numerator and denominator of (4), so that risk evaluations are based only on the likelihood function for $\mathbf{Z}$. We discuss evaluation of other prior distributions in the illustrative application.

For risk assessment for $T_{i j}$ in (5), we use a similar importance sampling approximation, resulting in

$$
q_{r}=\frac{\frac{\sum_{g=1}^{F} \pi_{g}^{(r)}\left\{\prod_{k=p+1}^{p+q} \lambda_{g g_{k}}^{(k)(r)}\left(\sum_{m=1}^{S} \omega_{g m}^{(r)} \prod_{k=1}^{p} \phi_{g m e}^{(k)(h)}\right)\right\}}{\sum_{g=1}^{F} \pi_{g}^{(r)}\left\{\prod_{k=p+1}^{p+q} \lambda_{g t_{i k}}^{(k)(h)}\left(\sum_{m=1}^{S} \omega_{g m}^{(r)} \prod_{k=1}^{p} \phi_{g m t_{i j k}}^{(k)(h)}\right)\right\}}}{\sum_{u=1}^{R}\left(\frac{\sum_{g=1}^{F} \pi_{g}^{(u)}\left\{\prod_{k=p+1}^{p+q} \lambda_{g c_{k}}^{(k)(u)}\left(\sum_{m=1}^{S} \omega_{g m}^{(u)} \prod_{k=1}^{p} \phi_{g m e j k}^{(k)(u)}\right)\right\}}{\sum_{g=1}^{F} \pi_{g}^{(u)}\left\{\prod_{k=p+1}^{p+q} \lambda_{g t_{i k}}^{(k)(u)}\left(\sum_{m=1}^{S} \omega_{g m}^{(u)} \prod_{k=1}^{p} \phi_{g m t_{i j k}}^{(k)(u)}\right)\right\}}\right)} .
$$

\section{Disclosure risk assessments for synthesis without structural zeros}

To evaluate the disclosure risks for individuals, we drop each individual record in $\mathbf{D}$ one at a time. For each individual $i j$, we compute the resulting $\rho_{i j}^{t}$ for all $t$ in the reduced support $\mathcal{R}_{i j}$. Here, each $\mathcal{R}_{i j}$ is the union of the true $t_{i j}$ plus the 39 other combinations of $t$ obtained by changing one variable in $t_{i j}$ to any possible outcome. For any two records $i j$ and $i^{\prime} j^{\prime}$ such that $t_{i j}=t_{i^{\prime} j^{\prime}}$ in $\mathbf{D}, \rho_{i j}^{t}=\rho_{i^{\prime} j^{\prime}}^{t}$ for any possible $t$. Thus, we need only compute the set of $\rho_{i j}^{t}$ for the 15280 combinations that appeared in $\mathbf{D}$. We use a uniform prior distribution over all $t \in \mathcal{R}_{i j}$, for each record $i j$.

Figure 1 displays the distribution of the rank of the true $t_{i j}$ for each of the 15280 combinations. Here, a rank equal to 1 means the true $t_{i j}$ has the highest probability of being the unknown $T_{i j}$, whereas a rank of 40 means the true $t_{i j}$ has the lowest 
probability of being $T_{i j}$. As evident in the figures, even armed with $\mathbf{D}_{-i j}$ the intruder gives the top rank to the true $t_{i j}$ for only 11 combinations. The intruder gives $t_{i j}$ a ranking in the top three for only 194 combinations. We note that, even though 12964 combinations were unique in $\mathbf{D}$, the NDPMPM synthesizer involves enough smoothing that we do not recover the true $t_{i j}$ in the overwhelming majority of cases.

Figure 2 displays a histogram of the corresponding probabilities associated with the true $t_{i j}$ in each of the 15280 combinations. The largest probability is 0.2360 . Only 1 probability exceeds 0.2 , and 40 probabilities exceed 0.1 . The majority of probabilities are in the 0.03 range. As we assumed a uniform prior distribution over the 40 possibilities in $R_{i j}$, the ratio of the posterior to prior probability is typically around one. Only a handful of combinations have ratios exceeding two. Thus, compared to random guesses over a close neighborhood of the true values, $\mathbf{Z}$ typically does not provide much additional information about $t_{i j}$. We also look at the disclosure risks for households. To do so, we drop each household record in $\mathbf{D}$ one at a time. For households of size 2, the reduced support $\mathcal{R}_{i}$ comprises the true $t_{i}$ plus the 56 other combinations of $t$ obtained by changing $t_{i}$ in one variable. For the household-level variables, we change the entire variable for all members of the household. For the individual-level variable, we change one variable for each individual as before. We need only compute $\rho_{i}^{t}$ for each of the 5375 combinations of households of size 2 that appear in $\mathbf{D}$. We use a uniform prior distribution over all $t \in \mathcal{R}_{i}$.

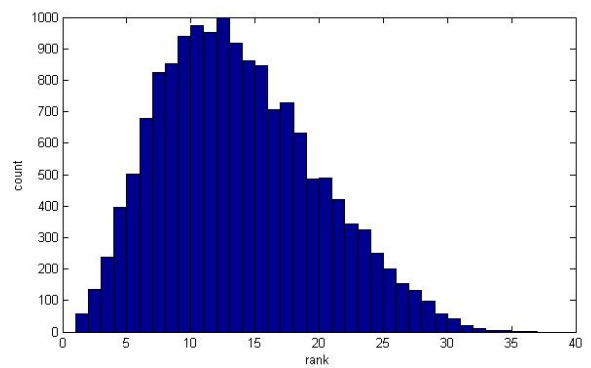

Figure 1: Histogram of ranks of the probabilities associated with true $t_{i j}$. Data have no structural zeros.

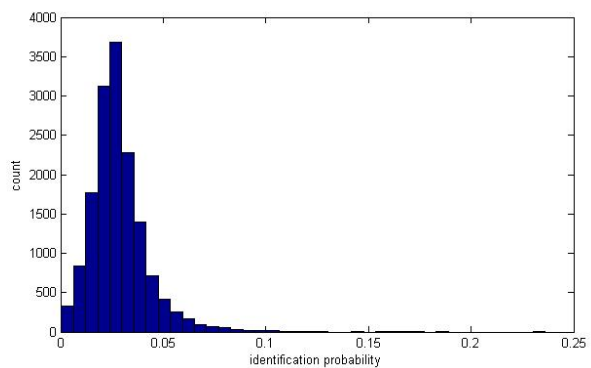

Figure 2: Histogram of re-normalized probabilities associated with the true $t_{i j}$. Data have no structural zeros.

Figure 3 displays the distribution of the rank of the true $t_{i}$ for each of the 5375 combinations. Once again, even armed with $\mathbf{D}_{-i}$, the intruder never gives the top rank to the true $t_{i}$. the intruder gives the true $t_{i}$ a ranking in the top three for only seven household combinations. We note that 5331 household combinations of size 2 were unique in $\mathbf{D}$. 


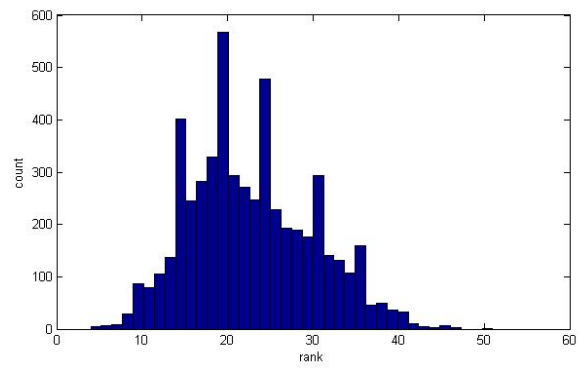

Figure 3: Histogram of ranks of the probabilities associated with true $t_{i}$, for households of size 2. Data have no structural zeros.

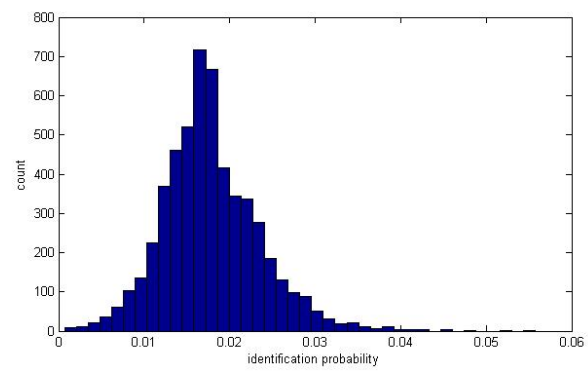

Figure 4: Histogram of re-normalized probabilities associated with the true $t_{i}$, for households of size 2. Data have no structural zeros.

Figure 4 displays a histogram of the corresponding probabilities associated with the true $t_{i}$ in each of the 5375 combinations of households of size 2 . The majority of probabilities are in the 0.02 range. As we assumed a uniform prior distribution over the 57 possibilities in the support, the ratio of the posterior to prior probability is typically around one. Thus, as with individuals, compared to random guesses over a close neighborhood of the true values, $\mathbf{Z}$ typically does not provide much additional information about $t_{i}$. The largest probability is 0.0557 .

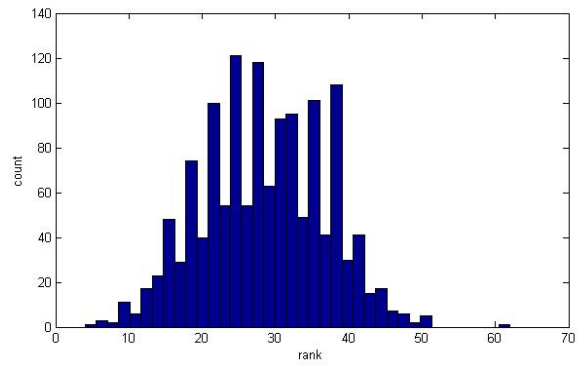

Figure 5: Histogram of ranks of the probabilities associated with true $t_{i}$, for households of size 3. Data have no structural zeros.

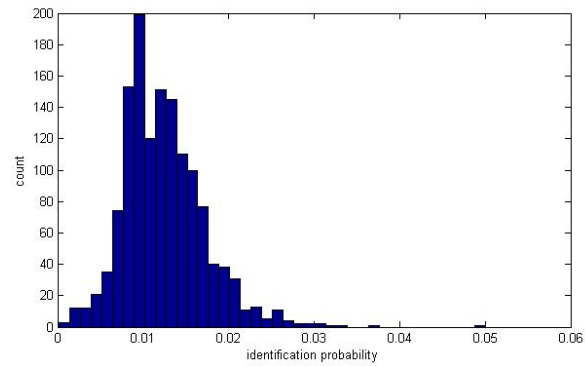

Figure 6: Histogram of re-normalized probabilities associated with the true $t_{i}$, for households of size 3. Data have no structural zeros.

For households of size 3 , the reduced support $\mathcal{R}_{i}$ comprises the true $t_{i}$ plus 81 other combinations of $t$ obtained by changing one variable at a time, as done for households of size 3 . We need only compute $\rho_{i}^{t}$ for each of the 1375 combinations that appear in $\mathbf{D}$. We use a uniform prior distribution over all $t \in \mathcal{R}_{i}$. 
Figure 5 displays the distribution of the rank of the true $t_{i}$ for each of the 1375 combinations. Even armed with $\mathbf{D}_{-i}$, the intruder gives $t_{i}$ a ranking in the top three for no combinations. We note that all these 1375 combinations were unique in $\mathbf{D}$, yet evidently the nested Dirichlet process synthesizer involves enough smoothing that we do not recover the true $t_{i}$ in the overwhelming majority of cases.

Figure 6 displays a histogram of the corresponding probabilities associated with the true $t_{i}$ in each of the 1375 combinations. The majority of probabilities are in the 0.010 range. As we assumed a uniform prior distribution over the 82 possibilities in the support, the ratio of the posterior to prior probability is typically one or less. Thus, compared to random guesses over a reasonably close neighborhood of the true values, $\mathbf{Z}$ typically does not provide much additional information about $t_{i}$. The largest probability is 0.0500 .

For households of size 4 , the reduced support $\mathcal{R}_{i}$ comprises the true $t_{i}$ plus 106 other combinations of $t$ obtained by changing one variable at a time, as with the other sizes. We do computations for each of the 478 combinations that appear in $\mathbf{D}$. We use a uniform prior distribution over all $t \in \mathcal{R}_{i}$.

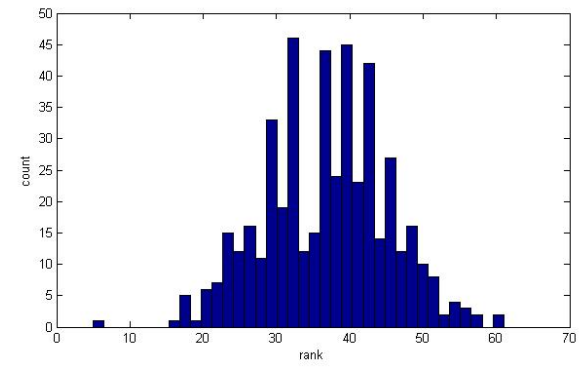

Figure 7: Histogram of ranks of the probabilities associated with true $t_{i}$, for households of size 4 . Data have no structural zeros.

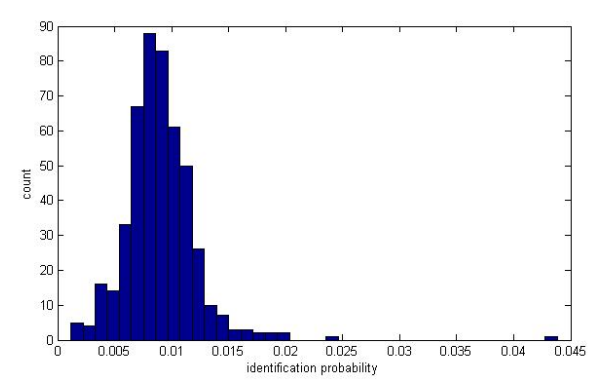

Figure 8: Histogram of re-normalized probabilities associated with the true $t_{i}$, for households of size 4. Data have no structural zeros.

Figure 7 displays the distribution of the rank of the true $t_{i}$ for each of the 478 combinations. The intruder gives the true $t_{i}$ a ranking in the top three for no combinations. All these 478 combinations were unique in D. Figure 8 displays a histogram of the corresponding probabilities associated with the true $t_{i}$ in each of the 478 combinations. The majority of probabilities are in the 0.01 range. As we assumed a uniform prior distribution over the 107 possibilities in the support, the ratio of the posterior to prior probability is typically one or less. Once again, $\mathbf{Z}$ typically does not provide much additional information about $t_{i}$. The largest probability is 0.0438 .

For households of size 5 , the reduced support $\mathcal{R}_{i}$ comprises the true $t_{i}$ plus 131 other combinations of $t$ obtained by changing one variable at a time, as with the other 
sizes. We do computations for each of the 123 combinations that appear in $\mathbf{D}$. We use a uniform prior distribution over all $t \in \mathcal{R}_{i}$.

Figure 9 displays the distribution of the rank of the true $t_{i}$ for each of the $123 \mathrm{com}-$ binations. The intruder gives the true $t_{i}$ a ranking in the top three for no combinations. All these 123 combinations were unique in $\mathbf{D}$. Figure 10 displays a histogram of the corresponding probabilities associated with the true $t_{i}$ in each of the 123 combinations. The majority of probabilities are in the 0.008 range. As we assumed a uniform prior distribution over the 132 possibilities in the support, the ratio of the posterior to prior probability is typically around one. Once again, $\mathbf{Z}$ typically does not provide much additional information about $t_{i}$. The largest probability is 0.0292 .

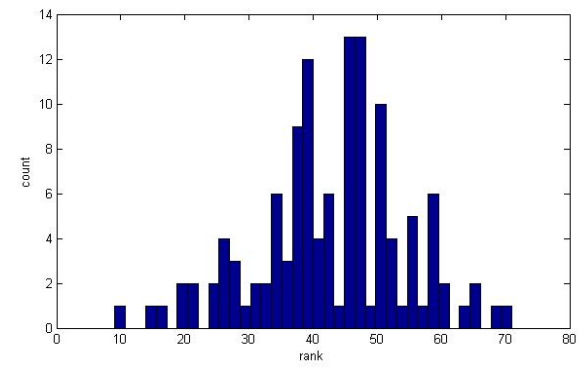

Figure 9: Histogram of ranks of the probabilities associated with true $t_{i}$, for households of size 5. Data have no structural zeros.

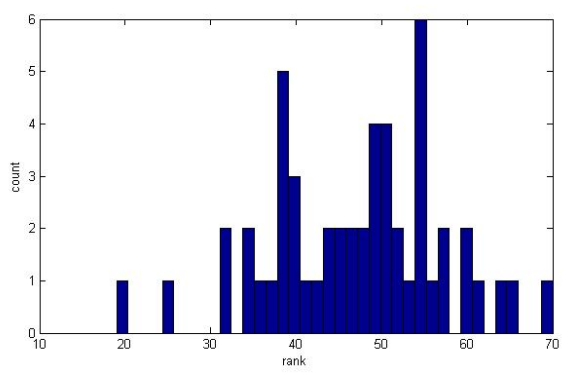

Figure 11: Histogram of ranks of the probabilities associated with true $t_{i}$, for households of size 6 . Data have no structural zeros.

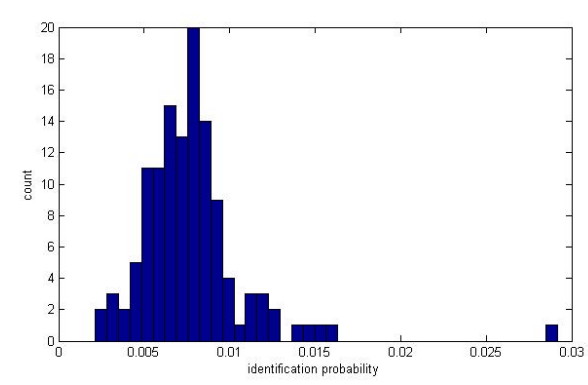

Figure 10: Histogram of re-normalized probabilities associated with the true $t_{i}$, for households of size 5. Data have no structural zeros.

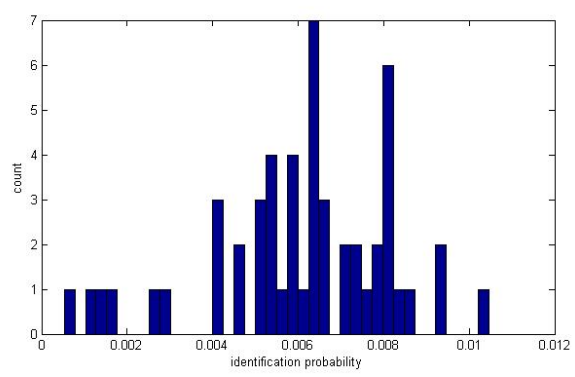

Figure 12: Histogram of re-normalized probabilities associated with the true $t_{i}$, for households of size 6. Data have no structural zeros.

For households of size 6 , the reduced support $\mathcal{R}_{i}$ comprises the true $t_{i}$ plus 156 
other combinations of $t$ obtained by changing one variable at a time, as with the other sizes. We do computations for each of the 52 combinations that appear in $\mathbf{D}$. We use a uniform prior distribution over all $t \in \mathcal{R}_{i}$.

Figure 11 displays the distribution of the rank of the true $t_{i}$ for each of the 52 combinations. The intruder gives the true $t_{i}$ a ranking in the top three for no combinations. All these 52 combinations were unique in $\mathbf{D}$. Figure 12 displays a histogram of the corresponding probabilities associated with the true $t_{i}$ in each of the 52 combinations. The majority of probabilities are in the 0.007 range. As we assumed a uniform prior distribution over the 157 possibilities in the support, the ratio of the posterior to prior probability is typically around one. Once again, $\mathbf{Z}$ typically does not provide much additional information about $t_{i}$. The largest probability is 0.0105 .

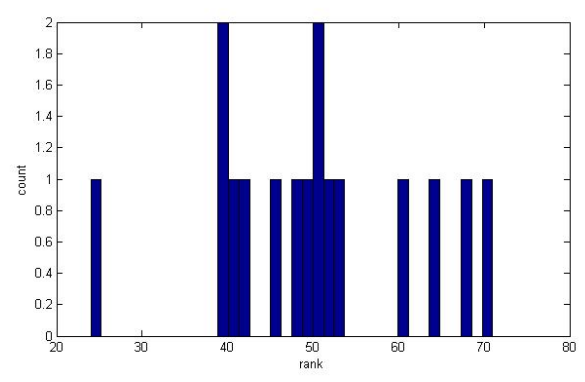

Figure 13: Histogram of ranks of the probabilities associated with true $t_{i}$, for households of size 7. Data have no structural zeros.

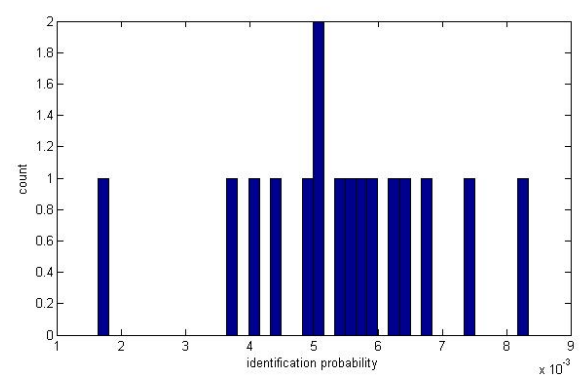

Figure 14: Histogram of re-normalized probabilities associated with the true $t_{i}$, for households of size 7. Data have no structural zeros.

For households of size 7 , the reduced support $\mathcal{R}_{i}$ comprises the true $t_{i}$ plus 181 other combinations of $t$ obtained by changing one variable at a time, as with the other sizes. We do computations for each of the 16 combinations that appear in $\mathbf{D}$. We use a uniform prior distribution over all $t \in \mathcal{R}_{i}$.

Figure 13 displays the distribution of the rank of the true $t_{i}$ for each of the 16 combinations. The intruder gives the true $t_{i}$ a ranking in the top three for no combinations. All these 16 combinations were unique in $\mathbf{D}$. Figure 14 displays a histogram of the corresponding probabilities associated with the true $t_{i}$ in each of the 16 combinations. The majority of probabilities are in the 0.005 range. As we assumed a uniform prior distribution over the 182 possibilities in the support, the ratio of the posterior to prior probability is typically around one. Once again, $\mathbf{Z}$ typically does not provide much additional information about $t_{i}$. The largest probability is 0.0083 .

For households of size 8 , the reduced support $\mathcal{R}_{i}$ comprises the true $t_{i}$ plus 206 other combinations of $t$ obtained by changing one variable at a time, as with the other sizes. We do computations for each of the 5 combinations that appear in $\mathbf{D}$. We use a uniform prior distribution over all $t \in \mathcal{R}_{i}$. 
The ranks of the true $t_{i}$ for each of the 5 combinations are $\{52,39,84,57,67\}$. The intruder gives the true $t_{i}$ a ranking in the top three for no combinations. We note that all 5 household combinations of size 8 were unique in $\mathbf{D}$. The corresponding probabilities associated with the true $t_{i}$ in each of the 4 combinations are $\{0.0057,0.0049,0.0043,0.0075$, $0.0041\}$. As we assumed a uniform prior distribution over the 207 possibilities in the support, the ratio of the posterior to prior probability is typically around one. Once again, $\mathbf{Z}$ typically does not provide much additional information about $t_{i}$. The largest probability is 0.0075 .

For households of size 9 , the reduced support $\mathcal{R}_{i}$ comprises the true $t_{i}$ plus 231 other combinations of $t$ obtained by changing one variable at a time, as with the other sizes. We do computations for each of the 2 combinations that appear in $\mathbf{D}$. We use a uniform prior distribution over all $t \in \mathcal{R}_{i}$.

The ranks of the true $t_{i}$ for each of the 2 combinations are $\{57,66\}$. We note that both 2 household combinations of size 9 were unique in $\mathbf{D}$. The corresponding probabilities associated with the true $t_{i}$ in each of the 2 combinations are $\{0.0029,0.0017\}$. As we assumed a uniform prior distribution over the 232 possibilities in the support, the ratio of the posterior to prior probability is less than one. Once again, $\mathbf{Z}$ typically does not provide much additional information about $t_{i}$.

\section{Disclosure risk assessments for structural zeros example}

We now turn to illustrating the assessment of disclosure risks for the synthesis with structural zeros, described in Section 4.2 of the main text. For individual disclosure risks, for each individual $i j$ we compute the $\rho_{i j}^{t}$ for all $t$ in $\mathcal{R}_{i j}$ defined as the union of the true $t_{i j}$ plus the 24 other combinations of $t$ obtained by changing one variable at a time, keeping the relationship variable fixed as a computational convenience. We compute $\rho_{i j}^{t}$ for each of the 2517 combinations that appear in $\mathbf{D}$. We use a uniform prior distribution over all $t \in \mathcal{R}_{i j}$.

Figure 15 displays the distribution of the rank of the true $t_{i j}$ for each of the 3517 combinations. Even armed with $\mathbf{D}_{-i j}$, the intruder gives the top rank to the true $t_{i j}$ for only 33 combinations. The intruder gives the true $t_{i j}$ a ranking in the top three for 269 combinations. We note that 1204 combinations were unique in $\mathbf{D}$.

Figure 16 displays a histogram of the corresponding probabilities associated with the true $t_{i}$ in each of the 3517 combinations. The majority of probabilities are in the 0.03 range. As we assumed a uniform prior distribution over the 25 possibilities in the support, the ratio of the posterior to prior probability is typically only slightly above one. Thus, compared to random guesses over a close neighborhood of the true values, $\mathbf{Z}$ typically does not provide much additional information about $t_{i j}$. The largest probability is 0.3878 , and only 4 probabilities exceed $0.3,27$ probabilities exceed 0.2 , and 183 probabilities exceed 0.1 .

We also look at the disclosure risks for households. For households of size 2, the reduced support $\mathcal{R}_{i}$ consists of the true $t_{i}$ plus 31 other combinations of $t$ obtained 
by changing $t_{i}$ in one variable. We need only do computations for each of the 4070 combinations that appeared in $\mathbf{D}$. We use a uniform prior distribution over all $t \in \mathcal{R}_{i}$.

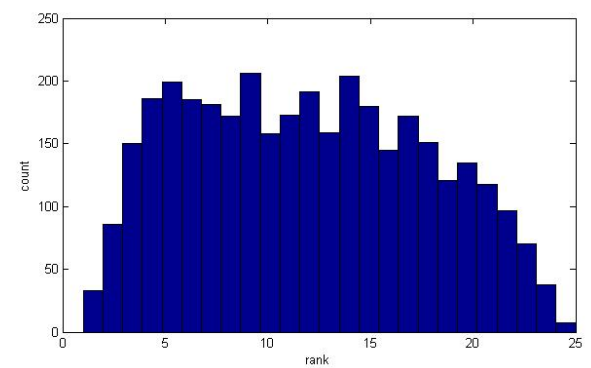

Figure 15: Histogram of ranks of the probabilities associated with true $t_{i j}$. Data have structural zeros.

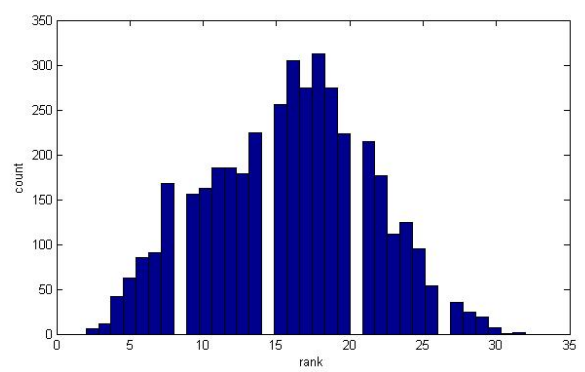

Figure 17: Histogram of ranks of the probabilities associated with true $t_{i}$, for households of size 2. Data have structural zeros.

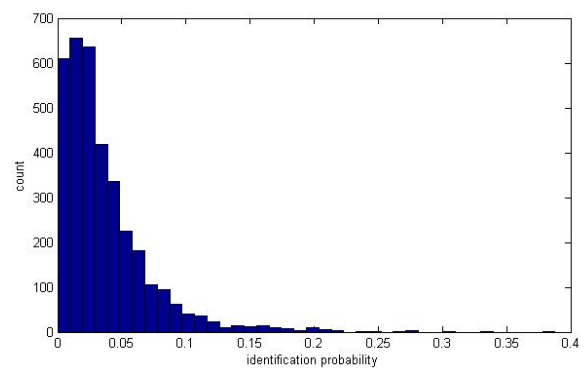

Figure 16: Histogram of re-normalized probabilities associated with the true $t_{i j}$, all individuals. Data have structural zeros.

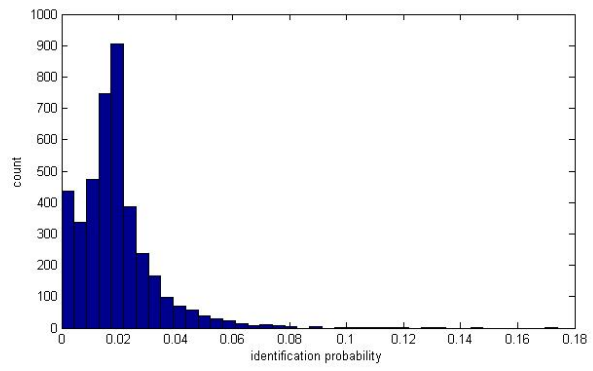

Figure 18: Histogram of re-normalized probabilities associated with the true $t_{i}$, for households of size 2. Data have structural zeros.

Figure 17 displays the distribution of the rank of the true $t_{i}$ for each of the 4070 combinations. Even armed with $\mathbf{D}_{-i}$, the intruder gives the top rank to the true $t_{i}$ for no household combination, and gives $t_{i}$ a ranking in the top three for only 18 combinations. We note that 3485 combinations were unique in $\mathbf{D}$.

Figure 18 displays a histogram of the corresponding probabilities associated with the true $t_{i}$ in each of the 4070 combinations. The majority of probabilities are in the 0.025 range. As we assumed a uniform prior distribution over the 32 possibilities in the support, the ratio of the posterior to prior probability is typically one or less. Thus, compared to random guesses over a reasonably close neighborhood of the true values, $\mathbf{Z}$ 
typically does not provide much additional information about $t_{i}$. The largest probability is 0.1740 , and only 15 probabilities exceed 0.1 .

For households of size 3 , the reduced support $\mathcal{R}_{i}$ consists of the true $t_{i}$ plus 46 other combinations of $t$. We need only do computations for each of the 2492 combinations that appeared in $\mathbf{D}$. We use a uniform prior distribution over all $t \in \mathcal{R}_{i}$.

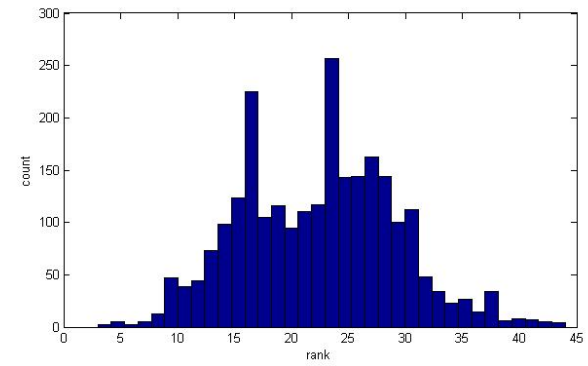

Figure 19: Histogram of ranks of the probabilities associated with true $t_{i}$, for households of size 3. Data have structural zeros.

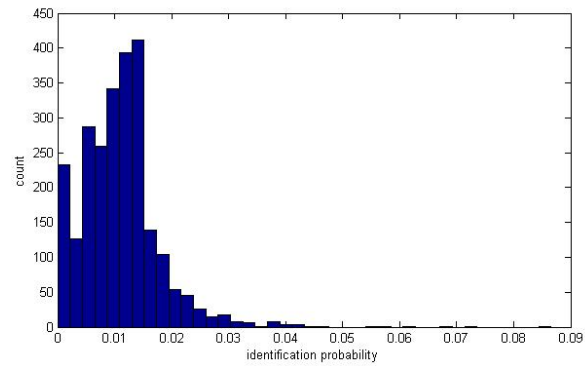

Figure 20: Histogram of re-normalized probabilities associated with the true $t_{i}$, for households of size 3. Data have structural zeros.

Figure 19 displays the distribution of the rank of the true $t_{i}$ for each of the 2492 combinations. Even armed with $\mathbf{D}_{-i}$, the intruder gives the top rank to the true $t_{i}$ for no combination and gives $t_{i}$ a ranking in the top three for only 2 combinations. We note that 2480 combinations were unique in $\mathbf{D}$.

Figure 20 displays a histogram of the corresponding probabilities associated with the true $t_{i}$ in each of the 2492 combinations. The majority of probabilities are in the 0.01 range. As we assumed a uniform prior distribution over the 47 possibilities in the support, the ratio of the posterior to prior probability is typically less than one. Thus, compared to random guesses over a reasonably close neighborhood of the true values, $\mathbf{Z}$ typically does not provide much additional information about $t_{i}$. The largest probability is 0.0866 .

For households of size 4 , the reduced support $\mathcal{R}_{i}$ consists of the true $t_{i}$ plus 61 other combinations of $t$. We need only do computations for each of the 2124 combinations that appeared in $\mathbf{D}$. We use a uniform prior distribution over all $t \in \mathcal{R}_{i}$.

Figure 21 displays the distribution of the rank of the true $t_{i}$ for each of the 2124 combinations. Even armed with $\mathbf{D}_{-i}$, the intruder gives the top rank to the true $t_{i}$ for no combination and gives $t_{i}$ a ranking in the top three for no combinations. We note that 2122 combinations were unique in $\mathbf{D}$.

Figure 22 displays a histogram of the corresponding probabilities associated with the true $t_{i}$ in each of the 2124 combinations. The majority of probabilities are in the 
0.01 range. As we assumed a uniform prior distribution over the 62 possibilities in the support, the ratio of the posterior to prior probability is typically less than one. Thus, compared to random guesses over a reasonably close neighborhood of the true values, $\mathbf{Z}$ typically does not provide much additional information about $t_{i}$. The largest probability is 0.0544 .

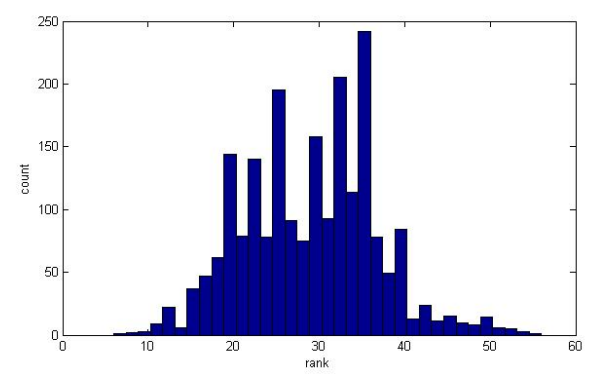

Figure 21: Histogram of ranks of the probabilities associated with true $t_{i}$, for households of size 4. Data have structural zeros.

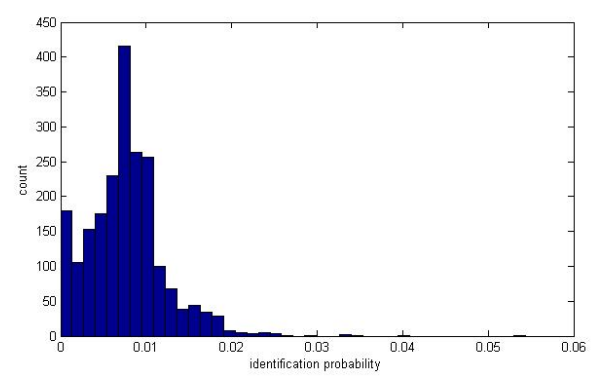

Figure 22: Histogram of re-normalized probabilities associated with the true $t_{i}$, for households of size 4. Data have structural zeros.

\section{Synthetic data and original sample estimates versus population values}

In this section, we present plots of point estimates for the original samples versus the values in the constructed populations, and for the synthetic data versus the values in the constructed populations. Figure 23 and Figure 24 display plots for the no structural zeros simulation described in Section 4.1 of the main text. Figure 25 and Figure 26 display plots for the structural zeros simulation described in the main text. In both simulation scenarios, the synthetic data and the original sample point estimates are close to the population values. 

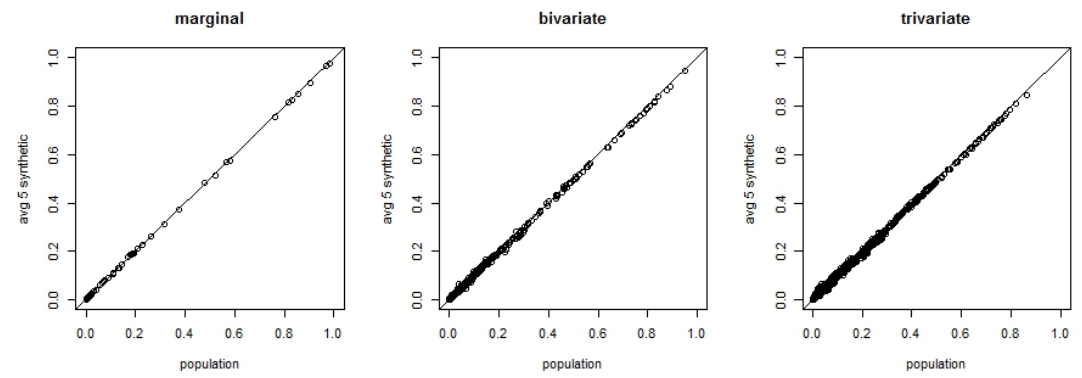

Figure 23: Marginal, bivariate and trivariate probabilities computed in the population and synthetic datasets for the illustration without structural zeros. Point estimates from the synthetic datasets and the population parameters are similar, suggesting that the NDPMPM estimates the population parameters well.
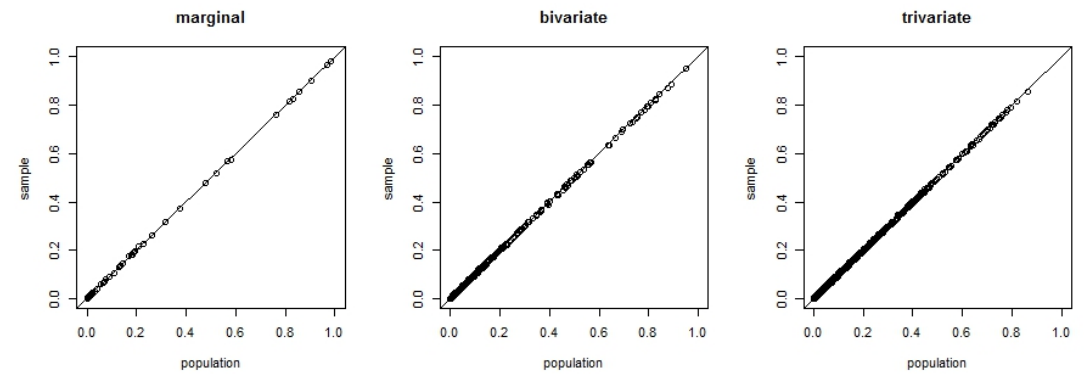

Figure 24: Marginal, bivariate and trivariate probabilities computed in the population and the sample for the illustration without structural zeros. Point estimates from the sample and the population parameters are similar. 

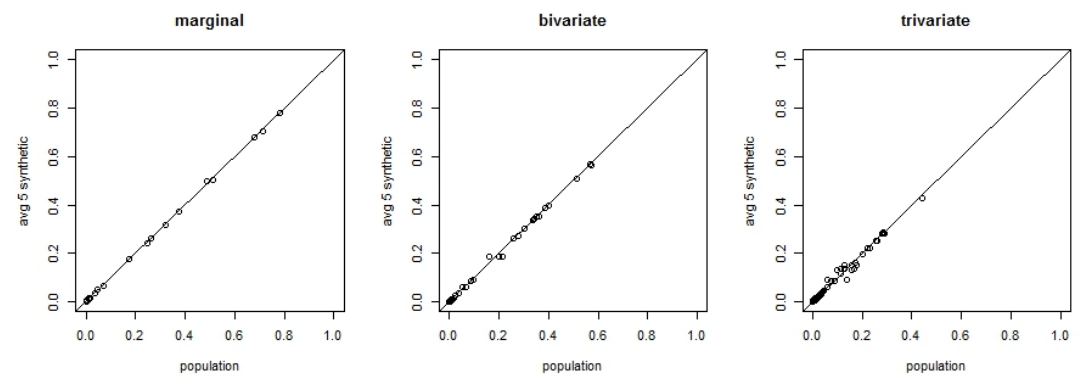

Figure 25: Marginal, bivariate and trivariate distributions probabilities computed in the population and synthetic datasets in illustration with structural zeros. Point estimates from the synthetic datasets and the population parameters are similar, suggesting that the NDPMPM estimates the population parameters well.
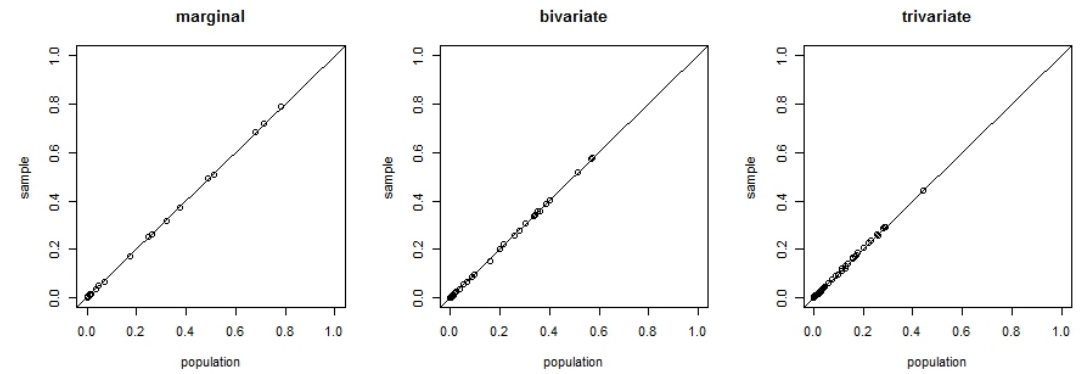

Figure 26: Marginal, bivariate and trivariate distributions probabilities computed in the population and the sample in illustration with structural zeros. Point estimates from the sample and the population parameters are similar.

\section{Uniform prior results in the no structural zeros simulation}

In the main text, we presented results based on using the empirical marginal frequencies as the shape parameters for the Dirichlet distributions in the main text. Here, we present results using uniform prior distributions for $\lambda$ and $\phi$ for the scenario with no structural zeros (Section 4.1 in the main text).

Figure 27 displays plots of point estimates with the uniform priors, which are very similar to the plots in Figure 1 in the main text based on the empirical priors. Table 1 displays probabilities for within-household relationships for the model with the uniform prior distribution, along with the results based on the empirical prior distribution for comparison. We find no meaningful differences between the two sets of results. 

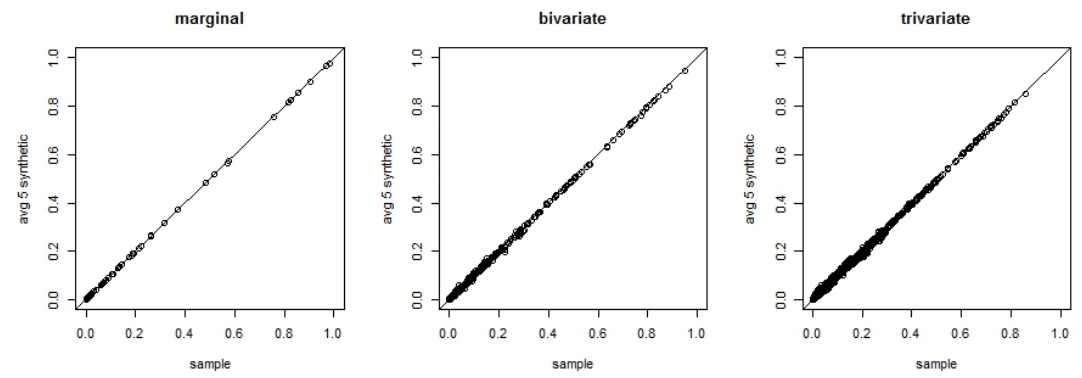

Figure 27: Marginal, bivariate and trivariate probabilities computed in the sample and synthetic datasets for the illustration without structural zeros, using uniform prior for $\lambda$ and $\phi$.

\begin{tabular}{lrrrr} 
& $\mathrm{Q}$ & Original & Uniform & Empirical \\
\hline All same race & & & & \\
$\quad n_{i}=2$ & .928 & $(.923, .933)$ & $(.840, .859)$ & $(.847, .868)$ \\
$n_{i}=3$ & .906 & $(.889, .901)$ & $(.809, .854)$ & $(.803, .845)$ \\
$\quad n_{i}=4$ & .885 & $(.896, .908)$ & $(.747, .831)$ & $(.730, .817)$ \\
All white and rent & .123 & $(.115, .128)$ & $(.110, .125)$ & $(.110, .126)$ \\
All white and have health coverage & .632 & $(.622, .641)$ & $(.579, .605)$ & $(.582, .603)$ \\
All married and working & .185 & $(.177, .192)$ & $(.163, .179)$ & $(.171, .188)$ \\
All have college degree & .091 & $(.086, .097)$ & $(.069, .080)$ & $(.071, .082)$ \\
All have health coverage & .807 & $(.800, .815)$ & $(.764, .784)$ & $(.764, .782)$ \\
All speak English & .974 & $(.969, .976)$ & $(.958, .966)$ & $(.959, .967)$ \\
Two workers in house & .291 & $(.282, .300)$ & $(.282, .304)$ & $(.289, .309)$ \\
\hline
\end{tabular}

Table 1: $95 \%$ confidence intervals in the original and synthetic data using a uniform prior and an empirical prior for selected probabilities that depend on within household relationships. Results for illustration without structural zeros. Intervals for probability that all family members are the same race are presented only for households of size two, three, and four because of inadequate sample sizes for $n_{i}>4$. The quantity $Q$ is the value in the constructed population of 308769 households.

\section{Results for larger number of components}

In this section, we present results using $(F, S)=(50,50)$ for the no structural zeros simulation, which results in many more classes than the results based on $(F, S)=$ $(30,10)$ that are presented in the main text. Figure 28 displays plots of point estimates with $(F, S)=(50,50)$. These are very similar to the plots in Figure 1 in the main text. Table 2 displays probabilities that depend on within-household relationships using these two sets of values of $(F, S)$. We find no meaningful differences between these two sets of results. 

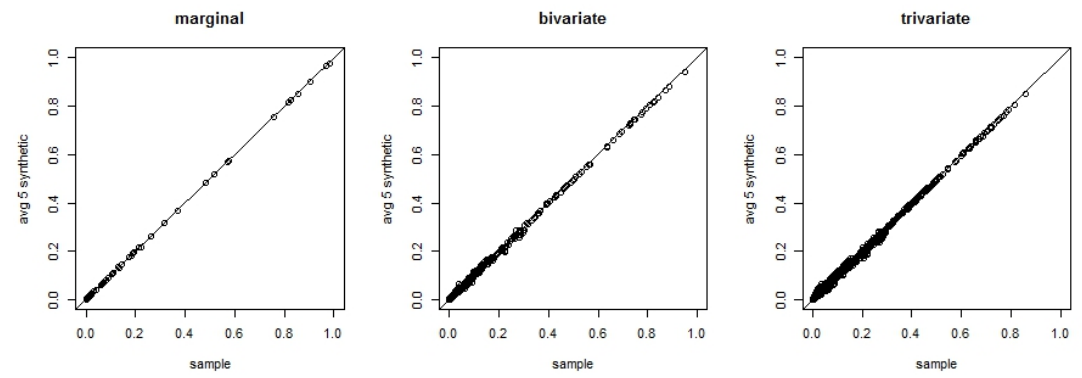

Figure 28: Marginal, bivariate and trivariate probabilities computed in the sample and synthetic datasets for the illustration without structural zeros, with $(F, S)=(50,50)$.

\begin{tabular}{lrrrr} 
& $\mathrm{Q}$ & Original & $(50,50)$ & $(30,10)$ \\
\hline All same race & & & & \\
$\quad n_{i}=2$ & .928 & $(.923, .933)$ & $(.835, .861)$ & $(.847, .868)$ \\
$\quad n_{i}=3$ & .906 & $(.889, .901)$ & $(.820, .861)$ & $(.803, .845)$ \\
$\quad n_{i}=4$ & .885 & $(.896, .908)$ & $(.755, .845)$ & $(.730, .817)$ \\
All white and rent & .123 & $(.115, .128)$ & $(.110, .125)$ & $(.110, .126)$ \\
All white and have health coverage & .632 & $(.622, .641)$ & $(.583, .606)$ & $(.582, .603)$ \\
All married and working & .185 & $(.177, .192)$ & $(.168, .186)$ & $(.171, .188)$ \\
All have college degree & .091 & $(.086, .097)$ & $(.069, .080)$ & $(.071, .082)$ \\
All have health coverage & .807 & $(.800, .815)$ & $(.761, .784)$ & $(.764, .782)$ \\
All speak English & .974 & $(.969, .976)$ & $(.958, .967)$ & $(.959, .967)$ \\
Two workers in house & .291 & $(.282, .300)$ & $(.291, .313)$ & $(.289, .309)$ \\
\hline
\end{tabular}

Table 2: $95 \%$ confidence intervals in the original and synthetic data (using $(F, S)=$ $(50,50)$ and $(F, S)=(30,10))$ for selected probabilities that depend on within household relationships. Results for illustration without structural zeros. Intervals for probability that all family members are the same race are presented only for households of size two, three, and four because of inadequate sample sizes for $n_{i}>4$. The quantity $Q$ is the value in the constructed population of 308769 households.

\section{References}

Dwork, C. (2006). "Differential privacy." In 33rd International Colloquium on Automata, Languages, and Programming, part II, 1-12. Berlin: Springer. 7

Hu, J., Reiter, J. P., and Wang, Q. (2014). "Disclosure risk evaluation for fully synthetic categorical data." In Domingo-Ferrer, J. (ed.), Privacy in Statistical Databases, 185199. Springer. 7,8

Manrique-Vallier, D. and Reiter, J. P. (2014). "Bayesian estimation of discrete multivariate latent structure models with strutural zeros." Journal of Computational and Graphical Statistics, 23: 1061 - 1079. 5 
Meng, X.-L. and Zaslavsky, A. M. (2002). "Single observation unbiased priors." The Annals of Statistics, 30: 1345-1375. 5

O'Malley, A. J. and Zaslavsky, A. M. (2008). "Domain-level covariance analysis for multilevel survey data with structured nonresponse." Journal of the American Statistical Association, 103: 1405-1418. 5 